\title{
Metastasectomy for Metastatic Renal Cell Carcinoma (mRCC): Lucky Break or Evidence-Based Approach?
}

\author{
Manuela Schmidinger, MD \\ Department of Medicine I, Clinical Division of Oncology and Comprehensive Cancer Center, Medical University of \\ Vienna, Vienna, Austria
}

In the last decade, physicians took a major step forward in the treatment of metastatic renal cell carcinoma (mRCC). Agents that target vascular endothelial growth factor receptor (VEGFR) signaling were shown to at least double overall survival (OS). However, complete remission (CR), long-term OS, and cure remain rare phenomena for patients with this disease. This may be explained in part by the fact that these agents exhibit their activity on the tumor vasculature instead of eradicating the cancer cell itself. Moreover, after a median treatment period of 11 months, tumors can overcome VEGFR inhibition by exploiting alternative pro-angiogenic pathways.

Findings show chemotherapy, as a strategy directed against the cancer cell, to be mostly ineffective in mRCC. ${ }^{1}$ In the past, durable CRs were occasionally achieved with high-dose interleukin-2, but few patients were found to be suitable for this procedure, and despite achievement of CR, cure remained anecdotal. However, to cure metastatic cancer, CR is a "minimum requirement."

Driven by the desire to achieve CR, surgical excision of metastases has been widely used in clinical practice, although only selected patients may derive a long-term benefit from this approach, particularly in the context of oligometastatic disease. Up to 200 retrospective reports have been published on this topic, primarily in the era of cytokine treatment. Almost all authors have reported a considerable survival benefit for patients undergoing

(C) Society of Surgical Oncology 2016

First Received: 4 September 2016;

Published Online: 26 September 2016

M. Schmidinger, MD

e-mail: manuela.schmidinger@meduniwien.ac.at metastasectomy. For instance, for patients undergoing complete resection of lung metastases, the 5-year survival period is shown to be 46.6 months. ${ }^{2}$ The authors have stated that the most favorable long-term OS would be achieved for patients with a long interval between diagnosis of the primary tumor and development of metastatic disease, unilateral metastases, and fewer than seven lesions in total. For patients with this profile, the OS is shown to be as long as 75.2 months.

The description of the patient-, tumor-, and disease-related factors used to identify patients who may benefit most from surgical excision of metastases already points toward the bias in these reports.

Patients with resectable (i.e., limited) metastatic disease who have a favorable prognosis and excellent performance status may achieve long-term survival anyway, and even more so currently in this era of multiple treatment options. Why then would we need another article suggesting the benefits of surgical excision?

In this issue, Brecheteau et al. ${ }^{3}$ report the results from a prospective database analysis of all $\mathrm{mRCC}$ patients treated at the University Hospital in Angers, France. Of 77 patients, $29 \%$ achieved CR, and $96 \%$ of these CR patients had undergone metastasectomy or percutaneous ablation, often multiple times. With an average CR duration of 18 months, the paper suggests that surgery remains an excellent option for mRCC, either to delay the start of firstline treatment (and resistance to first-line treatment) or to achieve long-term survival, with even the hope of cure for those without recurrence. The majority of publications on this topic in the past included patients from the cytokine era. However, this paper reports on outcomes from patients treated with surgery, targeted agents, or both.

Is this just another hypothesis-generating article with a low level of evidence? Scientifically, maybe yes, but even with the most conservative view of the results shown here 
and data published earlier, it must be acknowledged that local treatment of metastases follows a pattern, one that may enable long-term survival. But to what extent is this really true, bearing in mind the most common criticism: the bias in patient selection?

It appears obvious at first sight that only the fittest patients with favorable prognostic features would be considered for local procedures. Frequently in clinical practice, however, this may not be the primary driver of the decision. Patients may be referred for metastasectomy because of symptomatic metastases or metastases that may represent a risk for organ function (e.g., bone lesions with risk of fracture, brain lesions, and the like). For instance, in a report by Vogl et al. ${ }^{4}$ from the cytokine era, $43.4 \%$ of 99 patients underwent metastasectomy for different metastatic sites. The authors analyzed the reasons for these treatment decisions. Interestingly, the most common reason (for $65 \%$ of the patients) was to preserve organ function (e.g., a bone lesion with risk for fracture) rather than to achieve CR surgically. Based on this intention, a complete resection was achieved for only $21 \%$ of the patients, and the majority of the patients had other metastatic sites remaining after surgery. Nevertheless, the authors found that metastasectomy, although incomplete in almost $80 \%$ of the patients, was an independent predictor of survival (hazard ratio [HR], 0.29).

More recently, Orlova et al. ${ }^{5}$ presented retrospective data on incomplete metastasectomy in patients undergoing first-line treatment with targeted agents (TAs). Of 147 patients treated with TAs in a first-line setting, 47 underwent incomplete resection of metastases, whereas the remaining 100 were treated with TAs alone. The median OS for the patients undergoing surgery in addition to TAs was considerably longer than for the patients treated with TAs alone (46 vs 31 months). Moreover, incomplete metastasectomy was associated with a significantly longer progression-free survival in first-line treatment $(p=0.018)$.

These positive effects might not be restricted to surgical procedures. Mark et al. ${ }^{6}$ retrospectively analyzed the outcomes for patients undergoing any type of local intervention (e.g., metastasectomy, radiofrequency ablation, stereotactic radiosurgery) in combination with tyrosine kinase inhibitor (TKI) treatment. Patients with favorable or intermediate risk profiles had a median OS of 94.2 months when undergoing local intervention plus TKI treatment $(n=48)$ compared with 31.5 months when treated with TKIs alone $(n=26)(\mathrm{HR}, 0.28 ; 95 \%$ confidence interval [CI], 0.13-0.59; $p<0.001$ ).

So how do we move on from here? Of course, the scientific community would welcome data generated in a prospective randomized trial of local interventions for mRCC. However, it appears difficult to conduct such a trial. An enormous number of patients would be required to stratify for all different patient-, tumor-, and disease-related characteristics. Moreover, in clinical practice, the most appropriate therapeutic decision needs to be made for the individual patient, and no one would deny a patient a local intervention for a skeletal metastasis with risk of fracture, a single brain lesion suitable for stereotactic radiation, or metastases in the intestines with a risk of gastrointestinal bleeding or bowel perforation just because the evidence level is low.

In the future, indications for surgery or other local treatments may be considered even more generously in clinical practice. For instance, it is completely unknown how to deal with mixed responses, which have been observed repeatedly in this era of TA and even more currently in the era of checkpoint inhibitors. This phenomenon is poorly understood and may in part be explained by intratumor heterogeneity. ${ }^{7}$ Additional factors contributing to this behavior may lie in the tumor microenvironment (e.g., lymph node metastases were shown to be associated with unfavorable outcomes compared with other metastatic sites). ${ }^{8}$ Is it appropriate to change a systemic treatment that controls the majority of all metastatic sites well just because of a new lesion or a single lesion that does not "behave"? It might be difficult to influence intratumor heterogeneity or the tumor microenvironment therapeutically. In this context, surgical and other local approaches may help to delay a change of systemic treatment by excision of metastases expressing unknown or non-druggable targets.

In summary, the article by Brecheteau et al. ${ }^{3}$ adds important information on the value of local treatments in this era of TAs and checkpoint inhibitors. The potential chance to derive tremendous benefit from this procedure should outweigh the lack of level 1 evidence.

\section{REFERENCES}

1. Yagoda A, Abi-Rached B, Petrylak D. Chemotherapy for advanced renal cell carcinoma: 1983-1993. Semin Oncol. 1995;22:42-60.

2. Hofmann HS, Neef $\mathrm{H}$, Krohe K, et al. Prognostic factors and survival after pulmonary resection of metastatic renal cell carcinoma. Eur Urol. 2005;48:77-81.

3. Brecheteau F, Carrouget J, Lebdai S, et al. How did we obtain complete remission in patients who had metastatic renal cancer using targeted therapies? Eur Urol Suppl. 2016;15:e531. doi:10. 1016/S1569-9056(16)60533-X.

4. Vogl UM, Zehetgruber H, Dominkus M, et al. Prognostic factors in metastatic renal cell carcinoma: metastasectomy as independent prognostic variable. Br J Cancer. 2006;95:691-8.

5. Orlova R, Borisov P, Karlov PA, et al. Efficacy of incomplete metastasectomy with targeted therapy in patients with metastatic renal cell carcinoma. J Clin Oncol. 2016;34 (suppl;abstr e16117).

6. Mark JR, Merwarth CA, Brent N, et al. Effect of local intervention on renal cell carcinoma metastases in patients treated with tyrosine 
kinase inhibitors (TKIs) stratified by IMDC risk groups. J Clin Oncol. 2016;34 (suppl; abstr e16101).

7. Gerlinger M, Rowan AJ, Horswell S, et al. Intratumor heterogeneity and branched evolution revealed by multiregion sequencing. N Engl J Med. 2012;366:883-92.
8. Grassi P, Verzoni E, Porcu L, et al. Targeted therapies in advanced renal cell carcinoma: the role of metastatic sites as a prognostic factor. Future Oncol. 2014;10:1361-72. 